\begin{tabular}{ccc}
\hline & $\begin{array}{c}\text { International Journal of Health Services } \\
\text { Research and Policy } \\
\text { www.dergipark.org.tr/ijhsrp }\end{array}$ \\
$\begin{array}{c}\text { INTESEG } \\
\text { ENGINETIONAL } \\
\text { SCIENCE AND } \\
\text { EDUCATION GROUP }\end{array}$ & e-ISSN: 2602-3482 & IJHSRP \\
\hline RESTHST
\end{tabular}

Research Article

\title{
PROSPECTIVE EVALUATION OF MEDIUM-TERM OUTCOMES OF PATIENTS UNDERGOING HYSTEROSCOPIC UTERINE SEPTUM RESECTION
}

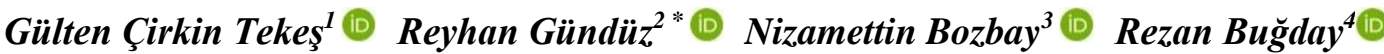 \\ Elif Ağaçayak ${ }^{2}$ (D)
}

\begin{abstract}
${ }^{1}$ Lice Halis Toprak Foundation State Hospital, Dep. of Obstetrics and Gynecology, Diyarbakır, Turkey ${ }^{2}$ Dicle University, Faculty of Medicine, Department of Obstetrics and Gynecology, Diyarbakır, Turkey

${ }^{3}$ Health Sciences University Gazi Yasargil Research and Training Hospital, Dep. of Obstetrics and Gynecology, Diyarbakır, Turkey

${ }^{4}$ Cizre Dr. Selahattin Cizrelioğlu State Hospital, Dep. of Obstetrics and Gynecology, Şırnak, Turkey Corresponding author: ryhn.gunduz@gmail.com
\end{abstract}

\begin{abstract}
The study aimed to retrospectively and prospectively investigate the pregnancy outcomes after hysteroscopy $(H / S)$ for septum resection in patients detected to have a uterine septum after presenting with a history of recurrent pregnancy loss (RPL), diagnosis of secondary infertility, or primary infertility. This study included 62 patients with infertility or a history of RPL who underwent hysteroscopic uterine septum resection. Patients who had uterine anomalies other than a uterine septum, such as submucous myoma, endometrial polyp were excluded. Those discharged home with a live infant after a uterine septum operation were compared based on certain variables, those who succeeded and those who failed were categorized into group 1 and group 2, respectively. The two groups were compared with regard to the total number of deliveries before and after the operation, the total number of abortions, preterm and term deliveries, delivery methods, control hysterosalpingographies (HSG), and whether they were reoperated. In our study, 25 (40\%) patients were primary infertile, $37(60 \%)$ had secondary infertility or a history of RPL. Hysteroscopic septum resection was performed under general anesthesia in 61 and spinal anesthesia in 1 of these patients. Of the patients who were postoperatively discharged home with a live infant, 8 (26.7\%) were determined to be primarily infertile and 22 (73.3\%) to have secondary infertility or a history of RPL. The rate of discharge with a live infant was determined as 76.9\% among patients who conceived. The success rate of Group 1 patients, who were secondary infertile or had a history of RPL, was significantly higher compared to patients who were primary infertile. Hysteroscopic uterine septum resection is a method with an easy application and high effectiveness. The success of the operation is particularly higher in patients who have a history of RPL or are secondary infertile compared with patients who are primary infertile.
\end{abstract}

Keywords: Infertility, recurrent pregnancy loss, uterine septum, hysteroscopy 


\section{Introduction}

Infertility is the inability to conceive despite no use of contraceptive methods at an appropriate frequency for one year or longer. Primary infertility is the absence of a history of pregnancy and secondary infertility is the absence of another pregnancy after having previously conceived by any method. The ability to conceive is defined as fecundability [1]. For a normal, young couple, the probability of conception is $25 \%$ at the end of one month and $85-90 \%$ at the end of one year. About 10 $15 \%$ of couples in the reproductive period complain of the inability to conceive [2]. The primary causes of infertility are; ovulatory dysfunction (20-40\%), tubal and peritoneal pathology (30-40\%), male factor (30-40\%), unexplained infertility (10\%), and uterine pathologies (10-15\%). Intrauterine pathologies can be surgically treated [3].

Recurrent pregnancy loss (RPL) is defined as two or more pregnancy losses. It affects less than $5 \%$ of couples. A significant portion is unexplained [4]. According to the Royal College of Obstetricians and Gynaecologists (RCOG) guidelines, the rate of Mullerian anomalies in individuals with RPL varies between $1.5 \%$ and $37 \%$ [5]. There is evidence suggesting that a uterine septum should be considered in RPL patients since uterine septa are associated with a higher number of pregnancy losses and uterine septum resection can decrease the rates of miscarriage [6].

Uterine septum is the most common congenital Mullerian anomaly [7]. It can contribute to the occurrence of unfavorable obstetric outcomes such as infertility, pregnancy loss and malpresentation, and preterm delivery. However, many women with uterine septa may not experience difficulty in reproduction [8]. There are a few minimally invasive surgical techniques used in uterine septum treatment. The primary therapeutic option for a uterine septum is septum resection with a resectoscope [9]. Septum resection was reported to be related to an improvement in the obstetric outcomes such as increasing the delivery rate, increasing the term delivery rate, and increasing the number of pregnancies in patients who were infertile prior to septum resection [10].

Our study aims to retrospectively evaluate the pregnancy outcomes after hysteroscopic septum resection in patients who presented to our clinic with a history of RPL, a diagnosis of secondary infertility or primary infertility, and were diagnosed with a uterine septum, and to contribute to the literature.

\section{Materials and Methods}

This study evaluated 62 patients who presented to the Gynecology and Obstetrics Clinic of Dicle University, Faculty of Medicine between January 2010-December 2018 with primary infertility, secondary infertility, or RPL, were diagnosed with septum based on hysterosalpingography (HSG) and ultrasonography (USG) and underwent hysteroscopic septum resection. Approval was obtained for this study from the local ethics committee of our University (Ethics committee Date:06.02.2020, No:103). Surgical consent forms included consent to the use of patients' information for the study.

All patients included in this study underwent HSG and USG. According to the examination results, patients with submucous myoma, endometrial polyp, and uterine anomalies other than a uterine septum (such as uterus didelphys, unicornuate uterus, bicornuate uterus) and patients diagnosed with infertility who had pathologies other than a uterine septum (such as an abnormal spermiogram, ovulatory dysfunction, tubal factor) were excluded from the study. Patients with complete or incomplete uterine septa were included in the study. 
According to the information in the hospital records, HSG was performed by measuring patients' uterine cavity length and injecting $1 \mathrm{cc}$ liquid contrast medium for each $1 \mathrm{~cm}$ of cavity length and each tube. USG was performed with a transabdominal and transvaginal approach. A 9-mm resectoscope with a 5-mm 30-degree optic and a bipolar or monopolar coagulation electrode was used. For diagnostic laparoscopy (L/S), 0-degree, two-dimensional L/S with white LED was used. All patients primarily underwent diagnostic $\mathrm{L} / \mathrm{S}$ and $\mathrm{H} / \mathrm{S}$ or only H/S. Patients who were found to have pathologies subsequently underwent operative H/S. During operative H/S, septum resection was performed on those with a septum. During the operation, resection of the septum was performed with scissors in 2 patients and using plasma kinetic energy with bipolar or monopolar coagulation electrodes with a resectoscope in 60 patients. The saline infusion was used as a distension medium. Patients were recommended to undergo a control HSG three months from the operation. Patients were postoperatively followed up for two years. Those who were discharged home with a live infant were compared based on certain variables, and those who succeeded and those who failed were categorized into group 1 and group 2, respectively. The two groups were compared with regard to demographic and clinical characteristics, type of infertility, and the presence of postoperative pregnancy.

Patients' phone numbers were obtained from the hospital information system. Patients were informed that the study did not involve any potentially harmful invasive interventions and that there would be no disclosure of information that could infringe on patient rights. The patients were asked questions regarding their age, gravidity, parity, abortion, number of living children, septum type, gestational week at delivery, history of surgery, method of delivery, and postoperative time to conception, and the responses were recorded.

The SPSS for Windows (version 16.0) program package was used for the statistical analyses. Data were tested for normal distribution using the Shapiro Wilk test. The Mann Whitney U test was used for the comparison of non-normally distributed variables in two independent groups. The chi-square test was used to investigate the relationships between categorical variables. Odds ratios and $95 \%$ confidence intervals were estimated. As descriptive statistics, quantitative variables were presented using mean \pm standard deviation, median (min-max) values, and the chi-square test was used for categorical variables. Statistically, $\mathrm{p}<0.05$ was considered significant.

\section{Results}

We identified 98 patients who presented to the Infertility Polyclinic of the Gynecology and Obstetrics Clinic of Dicle University, Faculty of Medicine between January 2010-December 2018 for primary infertility, secondary infertility, or RPL, were diagnosed with a uterine septum by HSG and/or USG and were operated. Sixty-two patients could be reached from their existing phone numbers and were enrolled in the study. Thirty-six patients could not be reached. Of the patients, 25 (40\%) were primary infertile and $37(60 \%)$ had a history of RPL or were secondary infertile. General anesthesia was induced in 61 patients as they were to undergo simultaneous L/S. Since one patient had previously undergone L/S, only H/S was performed under spinal anesthesia. Patients' mean age was $28.32 \pm 6.86$. Mean gravidity was determined as $2.53 \pm 2.27$.

Postoperatively, $39(62.9 \%)$ of the 62 patients conceived and $23(37.1 \%)$ did not. Of the 39 conceptions, $6(15.4 \%)$ were determined to result in spontaneous abortion, $6(15.4 \%)$ in preterm delivery, and 27 (69.2\%) in term delivery. All cases of abortion were observed to occur in the first 
trimester. No fetal anomalies were detected in $36(92.4 \%)$ of the achieved pregnancies, while anomalies were detected in $3(7.6 \%)$. Of the infants with anomalies, 2 showed cardiac anomalies and 1 showed hypospadias. The mean age of the patients who delivered infants with anomalies was determined as 28.33. One patient was determined to be primary infertile and 2 to be secondary infertile (Table 1 ).

Table 1. Evaluation of patients' data in frequency and percentages

\begin{tabular}{|c|c|c|c|}
\hline & & $\mathrm{n}$ & Percentage $\%$ \\
\hline \multirow[t]{2}{*}{ Uterine septum } & Complete septum & 6 & $9.6 \%$ \\
\hline & Incomplete septum & 56 & $90.4 \%$ \\
\hline \multirow{2}{*}{$\begin{array}{l}\text { Postoperative } \\
\text { pregnancy }\end{array}$} & No & 23 & $37.1 \%$ \\
\hline & Yes & 39 & $62.9 \%$ \\
\hline \multirow{3}{*}{$\begin{array}{l}\text { Postoperative } \\
\text { gestational week at } \\
\text { birth }\end{array}$} & Term & 27 & $69.2 \%$ \\
\hline & Preterm & 6 & $15.4 \%$ \\
\hline & Abortion & 6 & $15.4 \%$ \\
\hline \multirow{2}{*}{$\begin{array}{l}\text { History of } \\
\text { complication } \\
\text { during the } \\
\text { operation }\end{array}$} & No & 60 & $96.8 \%$ \\
\hline & Yes & 2 & $3.2 \%$ \\
\hline \multirow{3}{*}{$\begin{array}{l}\text { The outcome of } \\
\text { postoperative } \\
\text { pregnancy }\end{array}$} & Abortion & 6 & $15.3 \%$ \\
\hline & Vaginal birth & 10 & $25.8 \%$ \\
\hline & Cesarean & 23 & $58.9 \%$ \\
\hline \multirow{2}{*}{$\begin{array}{l}\text { Patients with fetal } \\
\text { anomalies }\end{array}$} & No & 36 & $92.4 \%$ \\
\hline & Yes & 3 & $7.6 \%$ \\
\hline \multirow{2}{*}{$\begin{array}{l}\text { History of } \\
\text { previous } \\
\text { abdominal surgery }\end{array}$} & No & 45 & $72.6 \%$ \\
\hline & Yes & 17 & $27.4 \%$ \\
\hline
\end{tabular}

Data are presented as percent

Patients who were discharged home with a live infant were assigned to group 1 and those who were not were assigned to group 2. In group 1, gravidity, parity, the number of living children were significantly higher $(\mathrm{p}<0.01)$. The number of abortions, age, and duration of marriage did not influence the success of the operation and no significant differences were found ( $p>0.05$ ) (Table 2).

Of the patients who were discharged home with a live infant, $8(26.7 \%)$ were determined to be primary infertile and $22(73.3 \%)$ to have a history of RPL or to be secondary infertile. In secondary infertility/RPL patient group, the rate of discharge with a live infant was 3.12 higher compared to the primary infertility group, and this difference was significant (OR:3.12, 95\% CI=1.07-9.05, p<0.05) (Table 3). In secondary infertility/RPL patients, the rate of pregnancies achieved in the postoperative period was 4.33 times higher compared with primary infertile patients, and this difference was significant $(\mathrm{p}<0.01)$. All patients whose pregnancies resulted in abortion were primary infertile. Compared with secondary infertile/RPL patients, the rate of abortion was significantly higher in primary infertile patients $(\mathrm{p}<0.01)$. 
Table 2. Comparison of patients' demographic and clinical characteristics between Group 1 and Group 2

\begin{tabular}{|c|c|c|c|c|c|c|c|}
\hline \multirow[b]{2}{*}{ Variables } & \multirow[b]{2}{*}{$\mathbf{n}$} & \multicolumn{2}{|c|}{ Group 1} & \multirow[b]{2}{*}{$\mathbf{n}$} & \multicolumn{2}{|c|}{ Group 2} & \multirow[b]{2}{*}{$\mathbf{p}$} \\
\hline & & $\operatorname{Mean} \pm$ SD & $\begin{array}{l}\text { Median } \\
{[\text { min-max] }}\end{array}$ & & $\operatorname{Mean} \pm$ SD & $\begin{array}{l}\text { Median } \\
{[\text { min-max] }}\end{array}$ & \\
\hline Gravidity & 30 & $3.43 \pm 2.16$ & $3\left[\begin{array}{ll}1 & -9\end{array}\right]$ & 32 & $1.69 \pm 2.07$ & $1[0-8]$ & $0.001 * *$ \\
\hline Parity & 30 & $1.8 \pm 1$ & $2[0-4]$ & 32 & $0.38 \pm 0.71$ & $0\left[\begin{array}{ll}0 & -3\end{array}\right]$ & $0.001 * *$ \\
\hline Abortion & 30 & $1.6 \pm 1.87$ & $1[0-7]$ & 32 & $1.31 \pm 1.64$ & $1[0-6]$ & 0.622 \\
\hline $\begin{array}{l}\text { Number of living } \\
\text { children }\end{array}$ & 30 & $1.73 \pm 0.91$ & $1.5[1-4]$ & 32 & $0.38 \pm 0.71$ & $0\left[\begin{array}{ll}0 & -3\end{array}\right]$ & $0.001 * *$ \\
\hline Age & 30 & $27.23 \pm 6.8$ & $28[2-42]$ & 32 & $29.34 \pm 6.87$ & $26.5[21-45]$ & 0.761 \\
\hline $\begin{array}{l}\text { Duration of } \\
\text { marriage (years) }\end{array}$ & 30 & $8.5 \pm 3.13$ & $8[3-14]$ & 32 & $9.25 \pm 6.08$ & $8[3-30]$ & 0.610 \\
\hline $\begin{array}{l}\text { Number of } \\
\text { postoperative } \\
\text { pregnancies }\end{array}$ & 30 & $1.6 \pm 0.89$ & $1[1-4]$ & 32 & $0.41 \pm 0.71$ & $0[0-2]$ & $0.001 * *$ \\
\hline $\begin{array}{l}\text { Number of septum } \\
\text { operations }\end{array}$ & 30 & $1.23 \pm 0.77$ & $1\left[\begin{array}{ll}1 & -5\end{array}\right]$ & 32 & $1.19 \pm 0.47$ & $1[1-3]$ & 0.818 \\
\hline $\begin{array}{l}\text { Gestational week at } \\
\text { birth }\end{array}$ & 30 & $37.47 \pm 2.49$ & 38 [28-41] & 9 & $18.44 \pm 15.27$ & $10[5-40]$ & $0.010 * *$ \\
\hline $\begin{array}{l}\text { Time to conception } \\
\text { (months) }\end{array}$ & 30 & $10.7 \pm 9.19$ & $10[1-36]$ & 9 & $12.67 \pm 15.01$ & $6[2-48]$ & 0.852 \\
\hline
\end{tabular}

$* \mathrm{p}<0.05 ; * \mathrm{p}<0.01$ statistically significant; Mann Whitney $\mathrm{U}$ test

Data are presented as mean $\pm \mathrm{SD}$

Table 3. Distribution of patients by type of infertility

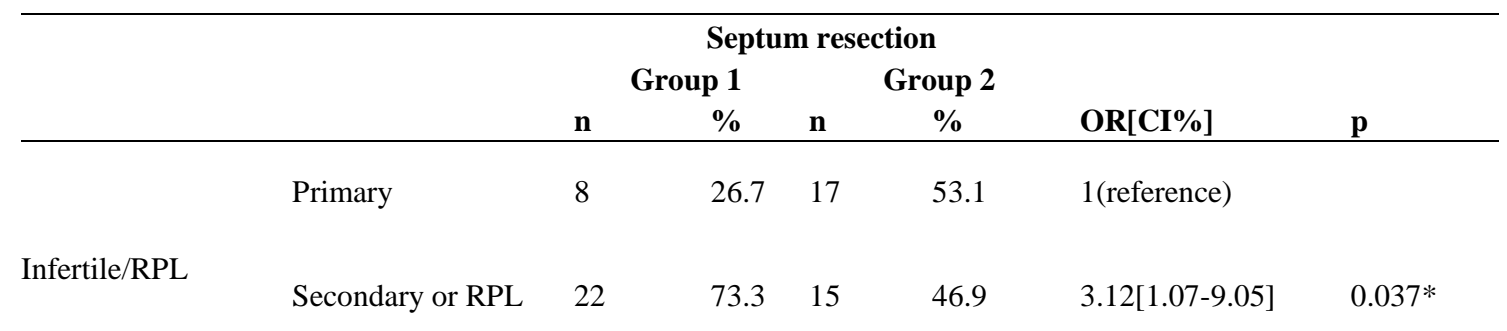

$*$ p $<0.05$ statistically significant; OR: Odds ratio, CI: Confidence interval RPL: recurrent pregnancy loss, Chi-square test

\section{Discussion}

We know that uterine septum is the most frequently encountered congenital Mullerian anomaly and that it can contribute to the occurrence of unfavorable obstetric outcomes such as infertility, pregnancy loss, and malpresentation, and preterm delivery [7,8]. In this study, we evaluated the rates of discharge with a live infant following hysteroscopic uterine septum resection in a patient group with infertility or RPL and determined that this operation is quite effective in the treatment.

Tonguc et al. reported in their study that all patients with a uterine septum who underwent hysteroscopic septum resection were operated on under general anesthesia and that all patients had undergone diagnostic L/S prior to H/S [7]. Paradisi et al. reported operating all patients under general anesthesia due to the concern that any one of the patients could require L/S [9]. In the present study, in 
agreement with the literature, 61 of the patients underwent operative $\mathrm{H} / \mathrm{S}$ with diagnostic L/S. One patient only underwent operative H/S. None of the patients underwent laparotomy.

To this date, many retrospective studies that evaluate pregnancy outcomes after hysteroscopic uterine septum resection have been published in the literature. The majority of these studies showed a postoperative improvement in pregnancy outcomes. Sendag et al. followed up patients with uterine septa who had a history of pregnancy for at least one year after hysteroscopic septum resection. They reported that, of the resulting pregnancies, 55\% were term deliveries, $10 \%$ were preterm deliveries, and $35 \%$ were spontaneous abortions [11]. Another study followed up 72 primary infertile patients with uterine septa for one year after uterine septum resection. Authors reported that 33 patients conceived; only $12 \%$ of these resulted in spontaneous abortions and 15\% resulted in preterm delivery [12]. Diverging from these two studies, our study included both primary infertile patients and patients with secondary infertility or RPL and conducted postoperative follow-up for two years. The results of our study are consistent with the literature; our live birth rate, which was $19.35 \%$ before the septum operation, increased from $0 \%$ to $26.7 \%$ in primary infertile patients, and from $29 \%$ to $73.3 \%$ in patients with secondary infertility or RPL. Since we determined that, 39 of the 62 patients in our study who conceived were able to conceive within an average period of 10 months, and that 30 of these (76\%) conceived within the first year, we recommend allowing at least one year for pregnancy. In our patients who conceived, we determined the rate of term delivery as $69.2 \%$, preterm delivery as $15.4 \%$, and total live birth as $76.9 \%$. The rate of abortion, which was $59.67 \%$ before the operation, decreased to $23.1 \%$ postoperatively. This situation in our study also clearly demonstrates that, in women with uterine septa and concomitant infertility or recurrent pregnancy loss, the most effective approach is hysteroscopic septum resection and that operation success is higher in patients with secondary infertility or RPL compared with primary infertile patients. This shows us that uterine septum causes pregnancies that result in abortion or preterm delivery rather than infertility.

Similar to our study, Gundabattula et al. evaluated primary and secondary infertile patients after uterine septum resection. They reported the rate of discharge with a live infant as $60 \%$ [13]. This study, the result of which is consistent with the rate we determined, differs from our study in that the number of patients with primary infertility is greater than that of patients with secondary infertility.

A study conducted by $\mathrm{Yu}$ et al. evaluated the occurrence of adhesion following septum resection. They categorized the patients into four groups as follows; postoperative estrogen therapy, intrauterine device use, intrauterine Foley use, and control. There was no significant difference between these four groups with regard to postoperative adhesion occurrence [14]. Upon review of the literature with regard to the occurrence of adhesion following uterine septum resection, there was no proven recommended treatment, and the hysteroscopic septum resection method was not recommended [15]. In our study, septum resection was performed on two patients using scissors, and on 60 patients using plasma kinetic energy with bipolar and monopolar coagulation electrodes with a resectoscope. Postoperatively, our patients were not given any treatment to prevent adhesion. Postoperative HSG was performed in 27 $(43.5 \%)$ patients and adhesion was not encountered.

Of the patients in our study, 6 (9.6\%) had a complete septum and 56 (90.4\%) had an incomplete septum. According to the literature, the width and length of the uterine septum are not correlated with pregnancy outcomes. ${ }^{15} \mathrm{We}$ are not able to make such a comment due to the low number of patients with a complete septum in our study; however, we think that more studies are needed. 
The limitations of our study are that not all of our patients underwent HSG in the postoperative period and that we were not able to reach all of the patients we had operated. The absence of data in the patient files regarding the size and width of the uterine septum constitutes another limitation of this study. On the other hand, the strength of our study is that all patients were operated at a tertiary hospital by doctors with at least 10 years of expertise.

\section{Conclusion}

Hysteroscopic uterine septum resection is an effective method with an easy application. Its success is particularly higher in patients with secondary infertility or a history of RPL compared to those with primary infertility. In this study, we determined a more significant improvement in the postoperative pregnancy outcomes of the group with secondary infertility or RPL. We recommend that primary infertile patients determined to have a uterine septum are operated but that other underlying causes are also investigated in patients who do not achieve pregnancy. A septum resection should be performed on patients with secondary infertility or a history of RPL and a period of at least one year should be allowed before the evaluation of treatment success.

Ethical Statement: An approval was obtained for this study from the local ethics committee of Dicle University Faculty of Medicine (Ethics committee's decision date and number: 06.02.2020; 103).

The compliance to Research and Publication Ethics: This work was carried out by obeying research and ethics rules.

\section{References}

[1] Schorge, O.J., Schaffer, I.J., Halvorson, M.L., Williams Gynecology, McGraw-Hill Professional, 2013. ISBN: 978-0-07-1472579.

[2] Boyar, H.I., "Female infertility and endocrinological diseases", Dicle Med J., 40(4), 700-703, 2013.

[3] Speroff, L., Fritz, M., Female infertility, Chapter 27, Clinical Gynecologic Endocrinology and Infertility, Eighth Edition, Lippincott Williams \& Wilkins, 2010.

[4] Li, Y.H., Marren, A., "Recurrent pregnancy loss: A summary of international evidence-based guidelines and practice”, Australian Journal of General Practice, 47(7), 432-436, 2018.

[5] The Investigation and Treatment of Couples with Recurrent First Trimester and Second Trimester Miscarriage, Royal College of Obstetricians and Gynaecologists, Guideline No. 17, London: RCOG, 2011.

[6] Practice Committee of the American Society for Reproductive Medicine. "Evaluation and treatment of recurrent pregnancy loss: A committee opinion”, Fertil Steril., 98(5), 1103-1111, 2012. Doi: 10.1016/j.fertnstert.2012.06.048.

[7] Tonguc, E.A., Var, T., Batioglu, S., "Hysteroscopic metroplasty in patients with a uterine septum and otherwise unexplained infertility", International Journal of Gynecology and Obstetrics, 113(2), 128-130, 2011. 
[8] Valle, R.F., Ekpo, G.E., "Hysteroscopic metroplasty for the septate uterus: review and metaanalysis", J Min Invas Gynecol., 20(1), 22-42, 2013.

[9] Paradisi, R., Barzanti, R., Natali, F., et al. "Metroplasty in a large population of women with septate uterus", J Minimal Invas Gynecol., 18(4), 449-54, 2011.

[10] Agostini, A., De Guibert, F., Salari, K., et al, “Adverse obstetric outcomes at term after hysteroscopic metroplasty”, J Minimal Invas Gynecol., 16(4), 454-7, 2009.

[11] Sendag, F., Mermer, T., Yucebilgin, S., et al., "Reproductive Outcomes After Hysteroscopic Metroplasty for Uterine Septum”, Clin Exp Obstet Gynecol., 37(4), 287-289, 2010.

[12] Dalal, R.J., Pai, H.D., Palshetkar, N.P., et al., "Hysteroscopic Metroplasty in Women With Primary Infertility and Septate Uterus: Reproductive Performance After Surgery", J Reprod Med., 57(1-2), 13-16, 2012.

[13] Gundabattula, S.R., Joseph, E., Marakani, L.R., et al., "Reproductive outcomes after resection of intrauterine septum", Journal of Obstetrics and Gynaecology, 34, 235-237, 2014.

[14] Yu, X., Yuhan, L., Dongmei, S., et al, "The Incidence of Post-Operative Adhesion Following Transection of Uterine Septum: A Cohort Study Comparing Three Different Adjuvant Therapies", Eur J Obstet Gynecol Reprod Biol., 201, 61-64, 2016.

[15] Practice Committee of the American Society for Reproductive Medicine.Uterine septum: a guideline, Fertility and Sterility, 106(3), 530-540, 2016. 